\title{
Soybean Sudden Death Syndrome Causal Agent Fusarium brasiliense Present in Michigan
}

\author{
Jie Wang, ${ }^{1}$ Hyunkyu Sang, ${ }^{1}$ Janette L. Jacobs, ${ }^{1}$ Kjersten A. Oudman, ${ }^{1}$ Linda E. Hanson, ${ }^{1,2}$ and Martin I. Chilvers ${ }^{1, \dagger}$ \\ ${ }^{1}$ Department of Plant, Soil, and Microbial Sciences, Michigan State University, East Lansing, MI 48824; and ${ }^{2}$ Sugar Beet and \\ Bean Research Unit, U.S. Department of Agriculture Agricultural Research Service, Michigan State University, East Lansing, \\ MI 48824.
}

\begin{abstract}
Sudden death syndrome (SDS), caused by members of Fusarium solani species complex (FSSC) clade 2, is a major and economically important disease in soybean worldwide. The primary causal agent of SDS isolated to date in North America has been F. virguliforme. In 2014 and 2016, SDS symptoms were found in two soybean fields located on the same farm in Michigan. Seventy Fusarium strains were isolated from roots of the SDS-symptomatic soybeans in two fields. Phylogenetic analysis of partial sequences of elongation factor- $1 \alpha$, the nuclear ribosomal DNA intergenic spacer region, and the RNA polymerase II beta subunit revealed that the primary FSSC species isolated was $F$. brasiliense (58 and $36 \%$ in each field) and the remaining Fusarium strains were identified as $F$. cuneirostrum, $F$. phaseoli, an undescribed Fusarium sp. from

FSSC clade 2, and strains in FSSC clade 5 and FSSC clade 11. Molecular identification was supported with morphological analysis and a pathogenicity assay. The soybean seedling pathogenicity assay indicated that $F$. brasiliense was capable of causing typical foliar SDS symptoms. Both root rot and foliar disease severity were variable by strain, just as they are in $F$. virguliforme. Both FSSC 5 and FSSC 11 strains were also capable of causing root rot, but SDS foliar symptoms were not detected. To our knowledge, this is the first report of $F$. brasiliense causing SDS in soybean in the United States and the first report of $F$. cuneirostrum, $F$. phaseoli, an as-yet-unnamed Fusarium sp., and strains in FSSC clade 5 and FSSC clade 11 associated with or causing root rot of soybean in Michigan.
\end{abstract}

For the first time in recent U.S. history, planted soybean [Glycine $\max ($ L.) Merr.] acres surpassed planted corn (Zea mays L.) in 2018, at 89.6 million acres compared with 89.1 million acres (National Agricultural Statistics Service 2018). More than $80 \%$ of the soybean acreage is cultivated in the upper Midwest, and more than 2 million acres of soybean were planted and harvested in Michigan with an estimated yield of 2.83 metric tons in 2016. Although there is a high level of production, this can be affected by several factors. For example, one of the most destructive diseases in soybean is sudden death syndrome (SDS), which is caused by four Fusarium species (F. virguliforme O'Donnell \& T. Aoki, F. brasiliense T. Aoki \& O'Donnell, $F$. crassistipitatum T. Aoki \& O'Donnell, and F. tucumaniae T. Aoki, O'Donnell, \& Y. Homma) that reside within clade 2 of the F. solani species complex (FSSC) (Aoki et al. 2003, 2005, 2012). Although SDS has been reported from all major soybeanproducing states in the United States to date except North Dakota (Hartman et al. 2015; O'Donnell et al. 2010; Rupe et al. 2001; Wang and Chilvers 2016), F. virguliforme is the only SDS-causal pathogen reported in the United States. In contrast, all four Fusarium species causing SDS have been found in South America, specifically in Argentina and Brazil (Aoki et al. 2003, 2005), and both $F$. virguliforme and $F$. brasiliense were reported as causal agents

Current address for J. Wang: Department of Plant Biology, Michigan State University, East Lansing, MI 48824.

${ }^{\dagger}$ Corresponding author: M. I. Chilvers; chilvers@msu.edu

J. Wang and H. Sang contributed equally to this work.

Funding: This study was supported in part by the Michigan Soybean Promotion Committee, the North Central Soybean Research Program, and Project GREEEN.

The author(s) declare no conflict of interest.

Accepted for publication 8 November 2018.

() 2019 The American Phytopathological Society responsible for SDS outbreaks in South Africa (Tewoldemedhin et al. 2014, 2017).

The first symptoms of soybean SDS occur on the roots, with symptoms of necrosis and tap root decay (Roy et al. 1997). Foliar symptoms generally develop during mid- to late reproductive growth stages. Symptomatic leaves demonstrate interveinal chlorosis and necrosis, and premature defoliation can result (Hartman et al. 2015). All Fusarium species causing SDS are soilborne and are capable of producing macroconidia and chlamydospores as their major means of survival in soil or in plant debris (Westphal et al. 2008). In addition to soybean, $F$. virguliforme has been reported to infect a wide range of asymptomatic host plants (Kolander et al. 2012). Infested soil or plant debris serves as a primary inoculum for the next growing season, and the movement of infested soil or plant debris can contribute to pathogen dispersal (Nakajima et al. 1993; Roy et al. 1997).

The sickle- or boat-shaped macroconidium is one of the key morphological features to identify Fusarium species to the genus level (Leslie and Summerell 2006). Colony and microscopic morphology can resolve Fusarium strains into species or species complex levels, and additional colony and host pathogenicity characteristics can delineate the Fusarium species into further groupings such as formae specialis (Aoki et al. 2014). However, because of the high morphological similarity within some groups (e.g., within clades of the FSSC), molecular sequence markers are required for confident species discrimination (Aoki et al. 2003; Leslie and Summerell 2006; O'Donnell 2000). One such recommended region is parsimony informative characters within ribosomal DNA (rDNA) intergenic spacer (IGS) multiple sequence alignments, which can resolve species in clade 2 of the FSSC (Aoki et al. 2003, 2005, 2012; O'Donnell et al. 2010).

Soybean SDS has become increasingly problematic and well established across the soybean production region in Michigan. F. virguliforme was initially reported in six Michigan counties (Chilvers and Brown-Rytlewski 2010) and has since been detected in 28 counties as of 2016 (Chilvers et al. 2016; Wang and Chilvers 2016). As part of a population genetics project, more than 500 strains of $F$. virguliforme were collected from across the United States from 2012 to 2014, including from Arkansas, Illinois, Kansas, and Michigan 
(Wang and Chilvers, unpublished data). F. virguliforme isolate identity was confirmed with species-specific polymerase chain reaction (PCR) and quantitative polymerase chain reaction (qPCR) assays (Wang et al. 2015). In one Michigan location, diseased soybean plants with typical SDS symptoms were collected, and slowgrowing Fusarium colonies, typical of FSSC clade 2 (Aoki et al. 2003), were isolated from roots. However, none of the isolates or root samples tested positive for $F$. virguliforme. Therefore, the objectives of this study were (i) to identify the Fusarium species causing SDS on soybean at this location and (ii) to confirm species identification using both morphological characteristics and molecular phylogenetics.

\section{Materials and Methods}

Isolation from soybean roots. SDS-symptomatic soybean plants were collected from two soybean fields located in Montcalm County, Michigan $\left(43^{\circ} 18^{\prime} 36.00^{\prime \prime} \mathrm{N},-85^{\circ} 09^{\prime} 0.00^{\prime \prime} \mathrm{W}\right)$. Fusarium strains were isolated from the root of SDS-symptomatic plants as described in Wang and Chilvers (2016). Briefly, taproots were vertically split into two or three pieces and then cut into 3- to 4-cm lengths. Root pieces were disinfested with $0.5 \%$ sodium hypochlorite for $3 \mathrm{~min}$, rinsed in sterile deionized water for $1 \mathrm{~min}$, and dried on sterilized paper towels. Three to four pieces of surface-disinfested root pieces were placed on $2 \%$ water agar amended with $300 \mathrm{mg}$ per liter of streptomycin and $15 \mathrm{mg}$ per liter of metalaxyl and incubated in the laboratory at room temperature $\left(21^{\circ} \mathrm{C}\right)$ and in ambient light, with $12 \mathrm{~h}$ of light and $12 \mathrm{~h}$ of darkness per day for 5 days to induce formation of sporodochia. Conidia masses were picked from the sporodochia using a sterile microbiological loop, spread on $3 \%$ water agar, and incubated at room temperature for 8 to $10 \mathrm{~h}$ in ambient light. A single germinated macroconidium from each plate was transferred to potato dextrose agar (PDA) (39 g per liter; Neogen, Lansing, MI). For longterm storage, spore suspensions were stored in $20 \%$ glycerol at $-80^{\circ} \mathrm{C}$ (Leslie and Summerell, 2006).

DNA extraction and PCR amplification. DNA from 70 Fusarium strains was extracted using the phenol-chloroform method according to Peever et al. (1999). PCR was performed in a DNA polymerase mixture (Phusion High-Fidelity; New England BioLabs, Ipswich, MA) containing 50 to $250 \mathrm{ng}$ of DNA from each strain to amplify partial sequences of translation elongation factor- $1 \alpha$ (EF-1 $\alpha$ ) for all strains, the nuclear rDNA IGS region for strains identified as FSSC clade 2, and the RNA polymerase II beta subunit $(R P B 2)$ for strains identified as FSSC 5 and 11. Two primer sets, NL11/CNS1 and EF1/EF2, were used for the IGS region and EF$1 \alpha$ amplification, respectively (Aoki et al. 2003; O'Donnell et al. 1998). Two previously published primer sets, $5 \mathrm{~F} 2 / 7 \mathrm{cR}$ and $7 \mathrm{cF} /$ $11 \mathrm{aR}$ (O'Donnell et al. 2007; Reeb et al. 2004), were used for RPB2 amplification. In addition, four new primer sets were used. These included the following forward $(\mathrm{F})$ and reverse $(\mathrm{R})$ primers: F1-RPB2 (TACACCTTTGCATCCACTCTTTC) and R1-RPB2 (CATACCTGGTTGTGATCGGGG); F1-1-RPB2 (CTGCAAGCTCCA CTGCT) and R1-1-RPB2 (GGGGTGACTACATATTGTTAGCC); F2-RPB2 (CATTCTTTACTACCCGCAAAAACC) and R2-RPB2 (AAGAACACCTGCGCTCGAA); and F2-1-RPB2 (GACAAACTACT CTCGCCGTATG) and R2-1-RPB2(CGACCATGTGACGGAGTC). These new primer sets were generated from consensus sequence by multiple sequence alignment of RPB2 of FSSC strains using MEGA version 6.06 (Tamura et al. 2013)

Sequence and phylogenetic analysis. PCR amplicons were purified with ExoSAP-IT (Affymetrix, Santa Clara, CA), and submitted to Macrogen Corporation (Rockville, MD) for sequencing using the aforementioned primer sets and internal IGS primers (Aoki et al. 2003). For phylogenetic analysis, EF-1 $\alpha$, IGS, and RPB2 sequences of reference FSSC strains were obtained from the National Center for Biotechnology Information GenBank. Multiple sequence alignments were performed using MUSCLE (Edgar 2004). For each molecular marker, nucleotide substitution models were tested using the modelTest function in the phangorn R package (Schliep 2011) prior to building the phylogenetic trees. The best nucleotide substitution model was determined based on Bayesian information criteria.
Phylogenetic trees were built using MrBayes version 3 (Ronquist and Huelsenbeck 2003). The Bayesian consensus phylogenetic trees were visualized using the ggtree $\mathrm{R}$ package (Yu et al. 2017). EF-1 $\alpha$, IGS, and RPB2 sequences of Michigan FSSC strains were deposited into GenBank (Table 1).

Examination of morphological characters. Fusarium strains were grown on PDA in $90-\mathrm{mm}$ plates for 13 days at $25^{\circ} \mathrm{C}$ in the dark. The colony phenotypes were observed and images of colonies were taken. To characterize the morphology of macroconidia, the strains were grown on Spezieller Nährstoffarmer agar (SNA) for 13 days (Leslie and Summerell, 2006). Macroconidia stained with 1\% safranin $\mathrm{O}$ solution were observed and photographed using a Leica DM5000 B microscope (Leica Microsystems, Buffalo Grove, IL) with a Leica DFC300FX digital camera. The size of 50 randomly selected macroconidia was measured using the Leica application suite (Leica Microsystems).

Pathogenicity tests. To determine the pathogenicity of $F$. brasiliense and other FSSC strains to soybeans, a seedling pathogenicity screen was performed using the soybean cultivar Sloan. The 17 strains used for pathogenicity tests were as follows: $F$. brasiliense (NRRL 22678, MIMtc-A8, MIMtc-C1, MIMtc-C9, and MIMtcC12), F. cuneirostrum (NRRL 31157 and MIMtc-C11), F. phaseoli (NRRL 31156 and MIMtc-A12), an as-yet-unnamed Fusarium sp. (MIMtc-A4 and MIMtc-B8), F. tucumaniae (NRRL 31096), F. virguliforme (NRRL 22292), FSSC 5 (MIMtc-A16FS and MIMtcC17FS), and FSSC 11 (MIMtc-B3FS and MIMtc-C13FS). NRRL strains were obtained from the Agricultural Research Service Culture Collection (Peoria, IL). Each strain was grown on Nash-Snyder medium (NSM) at room temperature for 14 days. An inoculum slurry of each strain was made by blending five colonized plates, five noncolonized NSM plates, and $500 \mathrm{ml}$ of sterile deionized water in a Waring Pro blender. A 100-ml aliquot of the inoculum slurry was poured into a spawn bag containing $1.6 \mathrm{~kg}$ of autoclaved sorghum grain. The inoculum was allowed to colonize the sorghum grain for 4 weeks at room temperature under ambient light conditions. Inoculum (12 g) of each strain was mixed with $250 \mathrm{ml}$ of medium vermiculite and placed into a 354-ml waxed paper cup (Solo, Lake Forest, IL) in which three 5-mm drainage holes had been made. An additional $70 \mathrm{ml}$ of medium vermiculite was added to the cup and six soybean seeds (Sloan) were sown in each cup and covered with an additional layer of $70 \mathrm{ml}$ of medium vermiculite. Cups containing no infested sorghum were used for the control. Foliar SDS disease severity (using a 1 to 9 scale, where 1 is a healthy plant, 2 to 5 indicate increasing severity of leaf interveinal chlorosis followed by necrosis, and 6 to 9 indicate increasing symptoms of defoliation and premature death) was rated 28 days after planting (growth stage: $\mathrm{V} 4)$ in a growth chamber $\left(20^{\circ} \mathrm{C}, 14 \mathrm{~h}\right.$ of light) according to the modified method in Wang et al. (2019). The SDS disease severity index (DIX) score that accounted for both severity (DS) and disease incidence $(\mathrm{DI})$ was calculated as follows: DIX $=\mathrm{DI} \times(\mathrm{DS} /$ maximum disease score $) \times 100$. Roots were harvested, washed, and rated for root rot using a scale from 1 to 7 (where 1 is a plant with healthy roots and 7 indicates roots that are pithy or hollow, and having 80 to $100 \%$ reduction in root mass). The root rot DIX score was calculated as follows: DIX $=$ DI $\times($ DS/maximum disease score $) \times 100$. Roots and shoots were dried for $48 \mathrm{~h}$ at $50^{\circ} \mathrm{C}$ and weighed for dry mass. The experiment was performed twice with five replicates. To assess differences among the results of SDS DIX scores (in percentages), values of the root rot DIX (in percentages), dry shoot mass (in grams), or dry root mass (in grams) caused by Fusarium strains, a mixed-model analysis of variance was performed using restricted maximum likelihood estimation. Species was treated as a fixed factor and strain nested within the interaction between species and replicate was used as a random factor. The Shapiro-Wilk test was conducted to test for normality (Shapiro and Wilk 1965). In addition, significant differences among the values of SDS DIX, values of root rot DIX, dry shoot mass, or dry root mass were separated using the Tukey honestly significant difference test for LSMeans $(\alpha=0.05)$. All statistical analyses were performed using JMP software (version 14.0; SAS Institute Inc., Cary, NC). 
To complete Koch's postulates, reisolation of the pathogens was conducted from one of the five replicates, using the protocol stated above. Identification of Fusarium strains was performed using morphological and molecular identification techniques as described above.

\section{Results}

Species identification and abundance. In total, 70 Fusarium strains were isolated from SDS-symptomatic soybean roots based on sporodochia formation and colony morphological characteristics. After colony and microscopic identification, 50 strains were putatively identified to be members of the FSSC clade 2, whereas the other 20 Fusarium strains were placed into the FSSC but not clade 2 , because the growth rate, colony color, and/or sporodochia formation were different.

Molecular phylogeny. Three molecular markers were used to identify Fusarium species recovered from soybean roots collected in Montcalm, Michigan. Phylogenetic analysis of EF-1 $\alpha$ sequences determined 50 strains to be within clade 2 of the FSSC and the other 20 strains belonged to clades 5 and 11 of the FSSC (Fig. 1). The EF- $1 \alpha$ sequence identified strains to the species complex level, but it did not resolve specific species, especially within clade 2 of the FSSC. Analysis of the rDNA IGS sequences identified most of the clade 2 FSSC strains to be $F$. brasiliense $(n=35)$, whereas the rest of strains were identified as $F$. cuneirostrum $(n=9), F$. phaseoli $(n=3)$, and an as-yet-unnamed Fusarium sp. $(n=3)$ as shown in
Figure 2. In addition to strains belonging to clade 2 of the FSSC, $F$. solani species from clade $5(n=6)$ and clade $11(n=14)$ were identified and resolved using phylogenetic analysis of RBP2 sequences (Fig. 3).

Morphological characterization. Colony morphology of FSSC clade 2 strains (MIMtc-A1, MIMtc-C11, MIMtc-A12, and MIMtcB8) grown on PDA for 13 days displayed undulating margins with variations in color of white to tints of yellow with grayish-blue and/or pink on the aerial hyphae. Orange exudates were observed as droplets on the surface of the colony of $F$. cuneirostrum strain MIMtc-C11 (Fig. 4, A to D). Colonies of fast-growing FSSC 5 (MIMtc-A16FS) and FSSC 11 (MIMtc-C13FS) on PDA for 7 days displayed entire margins and were white (Fig. 4, E and F). Sporodochia of FSSC strains on SNA were yellowish-white to pale yellow, and macroconidia were falcate, mostly 3-5 septate, apex rounded, or a distinct sickle-shape with a notched foot cell (Fig. 4, G to L). The sizes of macroconidia were as follows: $F$. brasiliense strain MIMtc-A1, 41.76 to $47.0 \times 5.4$ to $6.5 \mu \mathrm{m}$ on average; $F$. cuneirostrum strain MIMtc-C11, 46.6 to $51.6 \times 5.3$ to $6.8 \mu \mathrm{m}$ on average; $F$. phaseoli strain MIMtc-A12, 40.4 to $46.7 \times 5.5$ to $6.8 \mu \mathrm{m}$ on average; Fusarium sp. strain MIMtc-B8, 48.6 to $53.2 \times 5.4$ to $7.0 \mu \mathrm{m}$ on average; FSSC 5 strain MIMtc-A16FS, 42.0 to $46.5 \times 5.3$ to $6.4 \mu \mathrm{m}$ on average; and FSSC 11 strain MIMtc-C13FS, 40.0 to $47.0 \times 5.4$ to $6.5 \mu \mathrm{m}$ on average.

Pathogenicity tests. Soybean inoculated with stains $F$. brasiliense NRRL 22678, MIMtc-C1, or F. tucumaniae NRRL 31096 showed

Table 1. Fusarium spp. strains isolated from sudden death syndrome-symptomatic soybean roots in Michigan from two different years with location and GenBank accession numbers of elongation factor $1 \alpha(\mathrm{EF}-1 \alpha)$, intergenic spacer (IGS), and RNA polymerase II beta subunit (RPB2) sequences ${ }^{\mathrm{y}}$

\begin{tabular}{|c|c|c|c|c|c|c|}
\hline \multirow[b]{2}{*}{ Strain } & \multirow[b]{2}{*}{ Species } & \multirow[b]{2}{*}{ Isolation year } & \multirow[b]{2}{*}{ Location } & \multicolumn{3}{|c|}{ GenBank accession number ${ }^{z}$} \\
\hline & & & & EF-1 $\alpha$ & IGS & RPB2 \\
\hline MIMtc-A1 & F. brasiliense & 2014 & Montcalm A & MH541870 & MH539737 & NA \\
\hline MIMtc-A13 & F. brasiliense & 2014 & Montcalm A & MH541882 & MH539734 & NA \\
\hline MIMtc-A17 & F. brasiliense & 2014 & Montcalm A & MH541857 & MH539733 & NA \\
\hline MIMtc-A3 & $F$. brasiliense & 2014 & Montcalm A & MH541858 & MH539732 & NA \\
\hline MIMtc-A7 & $F$. brasiliense & 2014 & Montcalm A & MH541886 & MH539729 & NA \\
\hline MIMtc-A8 & F. brasiliense & 2014 & Montcalm A & MH541856 & МH539728 & NA \\
\hline MIMtc-A9Brn & F. brasiliense & 2014 & Montcalm A & MH541914 & MH539727 & NA \\
\hline MIMtc-A9Wht & F. brasiliense & 2014 & Montcalm A & MH541916 & MH539726 & NA \\
\hline MIMtc-B1 & F. brasiliense & 2014 & Montcalm A & MH541868 & MH539725 & NA \\
\hline MIMtc-B11 & F. brasiliense & 2014 & Montcalm A & MH541871 & MH539724 & NA \\
\hline MIMtc-B13 & $F$. brasiliense & 2014 & Montcalm A & MH541913 & MH539723 & NA \\
\hline MIMtc-B14Blu & F. brasiliense & 2014 & Montcalm A & MH541891 & MH539721 & NA \\
\hline MIMtc-B15 & F. brasiliense & 2014 & Montcalm A & MH541873 & MH539720 & NA \\
\hline MIMtc-B5 & $F$. brasiliense & 2014 & Montcalm A & MH541899 & MH539718 & NA \\
\hline MIMtc-B6 & F. brasiliense & 2014 & Montcalm A & MH541854 & MH539717 & NA \\
\hline MIMtc-B7 & F. brasiliense & 2014 & Montcalm A & MH541915 & MH539716 & NA \\
\hline MIMtc-B9Blu & F. brasiliense & 2014 & Montcalm A & MH541894 & MH539714 & NA \\
\hline MIMtc-B9Brn & F. brasiliense & 2014 & Montcalm A & MH541866 & MH539713 & NA \\
\hline MIMtc-C1 & F. brasiliense & 2014 & Montcalm A & MH541906 & MH539712 & NA \\
\hline MIMtc-C10 & $F$. brasiliense & 2014 & Montcalm A & MH541904 & MH539711 & NA \\
\hline MIMtc-C12 & F. brasiliense & 2014 & Montcalm A & MH541872 & MH539709 & NA \\
\hline MIMtc-C15 & F. brasiliense & 2014 & Montcalm A & MH541877 & MH539708 & NA \\
\hline MIMtc-C2 & F. brasiliense & 2014 & Montcalm A & MH541905 & MH539707 & NA \\
\hline MIMtc-C3Brn & F. brasiliense & 2014 & Montcalm A & MH541889 & MH539706 & NA \\
\hline MIMtc-C6 & F. brasiliense & 2014 & Montcalm A & MH541901 & MH539705 & NA \\
\hline MIMtc-C9 & $F$. brasiliense & 2014 & Montcalm A & MH541885 & MH539704 & NA \\
\hline MIMtc-A6 & F. cuneirostrum & 2014 & Montcalm A & MH541853 & MH539730 & NA \\
\hline MIMtc-A11 & F. cuneirostrum & 2014 & Montcalm A & MH541897 & MH539736 & NA \\
\hline MIMtc-C11 & F. cuneirostrum & 2014 & Montcalm A & MH541850 & MH539710 & NA \\
\hline MIMtc-A12 & $F$. phaseoli & 2014 & Montcalm A & MH541881 & MH539735 & NA \\
\hline MIMtc-B4 & $F$. phaseoli & 2014 & Montcalm A & MH541910 & MH539719 & NA \\
\hline MIMtc-A4 & Fusarium sp. & 2014 & Montcalm A & MH541883 & MH539731 & NA \\
\hline \multirow[t]{2}{*}{ MIMtc-B14 } & Fusarium sp. & 2014 & Montcalm A & MH541912 & MH539722 & NA \\
\hline & & & & & \multicolumn{2}{|c|}{ (Continued on next page } \\
\hline
\end{tabular}

y The rDNA IGS and/or the RPB2 gene was not sequenced for all strains because of their ability to resolve species identification in clade 2 and other clades of the F. solani species complex (FSSC).

${ }^{\mathrm{z}}$ NA indicates that sequences were not used in molecular phylogenetics and thus were not obtained. 
severe interveinal chlorosis and necrosis on leaves, typical of SDS. $F$. phaseoli strain MIMtc-A12 and Fusarium sp. strain MIMtc-A4 also caused foliar chlorosis but not typical SDS symptoms. All strains of FSSC clade 2 ( F. brasiliense, F. cuneirostrum, F. phaseoli, an as-yetunnamed Fusarium sp., F. tucumaniae, and $F$. virguliforme) caused more root rot symptoms compared with the FSSC 5 strains and the noninoculated control. Strains of FSSC 5 and FSSC 11 (MIMtcA16FS, MIMtc-C17FS, and MIMtc-C13FS) caused moderate root rot ( $\sim 50$ to $60 \%$ root rot), except for strain MIMtc-B3FS (FSSC $11)$, which was more aggressive ( $88 \%$ root rot). All strains significantly reduced soybean dry shoot and root mass compared with the noninoculated control. Strains MIMtc-A12 (F. phaseoli), MIMtcC1 (F. brasiliense), MIMtc-C11 (F. cuneirostrum), NRRL 22678 (F. brasiliense), and MIMtc-B8 (an as-yet-unnamed Fusarium sp.) caused more than $83 \%$ shoot mass reduction compared with the noninoculated control. Strains MIMtc-A12 ( $F$. phaseoli), NRRL 22678 (F. brasiliense), MIMtc-C11 (F. cuneirostrum), and MIMtc-C1 (F. brasiliense) reduced root mass by $79 \%$ compared with the noninoculated control (Table 2). Koch's postulates were completed by reisolating Fusarium strains from the diseased roots and confirming their identity.

\section{Discussion}

In North America, $F$. virguliforme has been known as the only causal pathogen of soybean SDS (Aoki et al. 2003; Hartman et al. 2015; Wang and Chilvers 2016). This study confirms F. brasiliense as an additional SDS-causal pathogen in the United States. F. brasiliense is known to cause SDS in South America and Africa (Aoki et al. 2005). This finding is significant because it will affect disease management strategies and soybean breeding efforts, as $F$. virguliforme is no longer the sole SDS-causal pathogen in the United States.

$F$. brasiliense causing soybean SDS was first reported in Brazil (Aoki et al. 2005) and recently in South Africa (Tewoldemedhin et al. 2017). Tewoldemedhin et al. (2017) speculated that the origin of $F$. brasiliense in South Africa was from infested soybeans possibly imported from South America. O'Donnell et al. (2010) also speculated that SDS-causing Fusarium species may have originated in South America. Although $F$. brasiliense has been primarily reported in South America until recently, the first isolation of $F$. brasiliense (strain NRRL 22678) in the United States was from an unknown host in California in 1993 (Aoki et al. 2005; O’Donnell et al. 2010; Rupe et al. 2001), which is 1 year after the first isolation of $F$. brasiliense in Brazil (Aoki et al. 2005). The presence of the species in California and Michigan may indicate that $F$. brasiliense was present prior to modern agriculture. Alternatively, although these Fusarium species have never been isolated from seed, seed lots have the potential to be contaminated by infested soil, potentially facilitating movement between continents.

Interestingly, recent surveys of dry bean root rots in Michigan have revealed a high proportion of $F$. brasiliense (Jacobs et al. 2018). The source of $F$. brasiliense, F. cuneirostrum, and $F$. phaseoli in this study might be attributable to rotation with dry beans

Table 1. (Continued from previous page)

\begin{tabular}{|c|c|c|c|c|c|c|}
\hline \multirow[b]{2}{*}{ Strain } & \multirow[b]{2}{*}{ Species } & \multirow[b]{2}{*}{ Isolation year } & \multirow[b]{2}{*}{ Location } & \multicolumn{3}{|c|}{ GenBank accession number ${ }^{\mathbf{Z}}$} \\
\hline & & & & EF-1 $\alpha$ & IGS & RPB2 \\
\hline MIMtc-B8 & Fusarium sp. & 2014 & Montcalm A & MH541890 & MH539715 & NA \\
\hline MIMtc-A16FS & FSSC 5 & 2014 & Montcalm A & MH541859 & NA & MH684734 \\
\hline MIMtc-B10 & FSSC 5 & 2014 & Montcalm A & MH541903 & NA & MH684731 \\
\hline MIMtc-B16FS & FSSC 5 & 2014 & Montcalm A & MH541862 & NA & MH684730 \\
\hline MIMtc-C17FS & FSSC 5 & 2014 & Montcalm A & MH541884 & NA & MH684729 \\
\hline MIMtc-A2FS & FSSC 11 & 2014 & Montcalm A & MH541867 & NA & MH684723 \\
\hline MIMtc-B12FS & FSSC 11 & 2014 & Montcalm A & MH541863 & NA & MH684732 \\
\hline MIMtc-B13FS & FSSC 11 & 2014 & Montcalm A & MH541898 & NA & MH684728 \\
\hline MIMtc-B2FS & FSSC 11 & 2014 & Montcalm A & MH541864 & NA & MH684726 \\
\hline MIMtc-B3FS & FSSC 11 & 2014 & Montcalm A & MH541865 & NA & MH684733 \\
\hline MIMtc-C13FS & FSSC 11 & 2014 & Montcalm A & MH541887 & NA & MH684727 \\
\hline MIMtc-C3FSwht & FSSC 11 & 2014 & Montcalm A & MH541860 & NA & MH684717 \\
\hline F-16-11 & F. brasiliense & 2016 & Montcalm B & MH541911 & MH539751 & NA \\
\hline F-16-20 & F. brasiliense & 2016 & Montcalm B & MH541875 & MH539754 & NA \\
\hline F-16-38 & F. brasiliense & 2016 & Montcalm B & MH541848 & MH539747 & NA \\
\hline F-16-39 & F. brasiliense & 2016 & Montcalm B & MH541902 & MH539752 & NA \\
\hline F-16-43 & F. brasiliense & 2016 & Montcalm B & MH541907 & MH539744 & NA \\
\hline F-16-51 & F. brasiliense & 2016 & Montcalm B & MH541878 & MH539743 & NA \\
\hline F-16-54 & F. brasiliense & 2016 & Montcalm B & MH541851 & MH539741 & NA \\
\hline F-16-58 & F. brasiliense & 2016 & Montcalm B & MH541849 & MH539739 & NA \\
\hline F-16-59 & F. brasiliense & 2016 & Montcalm B & MH541892 & MH539738 & NA \\
\hline F-16-22 & F. cuneirostrum & 2016 & Montcalm B & MH541855 & MH539753 & NA \\
\hline F-16-30 & F. cuneirostrum & 2016 & Montcalm B & MH541895 & MH539749 & NA \\
\hline F-16-31 & F. cuneirostrum & 2016 & Montcalm B & MH541909 & MH539748 & NA \\
\hline F-16-40 & F. cuneirostrum & 2016 & Montcalm B & MH541908 & MH539746 & NA \\
\hline F-16-42 & F. cuneirostrum & 2016 & Montcalm B & MH541893 & MH539745 & NA \\
\hline F-16-52 & F. cuneirostrum & 2016 & Montcalm B & MH541880 & MH539742 & NA \\
\hline F-16-16 & F. phaseoli & 2016 & Montcalm B & MH541874 & MH539750 & NA \\
\hline F-16-21 & FSSC 5 & 2016 & Montcalm B & MH541900 & NA & MH684736 \\
\hline F-16-33 & FSSC 5 & 2016 & Montcalm B & MH541876 & NA & MH684735 \\
\hline F-16-8 & FSSC 11 & 2016 & Montcalm B & MH541869 & NA & MH684719 \\
\hline F-16-48 & FSSC 11 & 2016 & Montcalm B & MH541896 & NA & MH684721 \\
\hline F-16-49 & FSSC 11 & 2016 & Montcalm B & MH541917 & NA & MH684725 \\
\hline F-16-61 & FSSC 11 & 2016 & Montcalm B & MH541888 & NA & MH684718 \\
\hline F-16-67 & FSSC 11 & 2016 & Montcalm B & MH541861 & NA & MH684720 \\
\hline F-16-69 & FSSC 11 & 2016 & Montcalm B & MH541852 & NA & MH684722 \\
\hline F-16-72 & FSSC 11 & 2016 & Montcalm B & MH541879 & NA & MH684724 \\
\hline
\end{tabular}




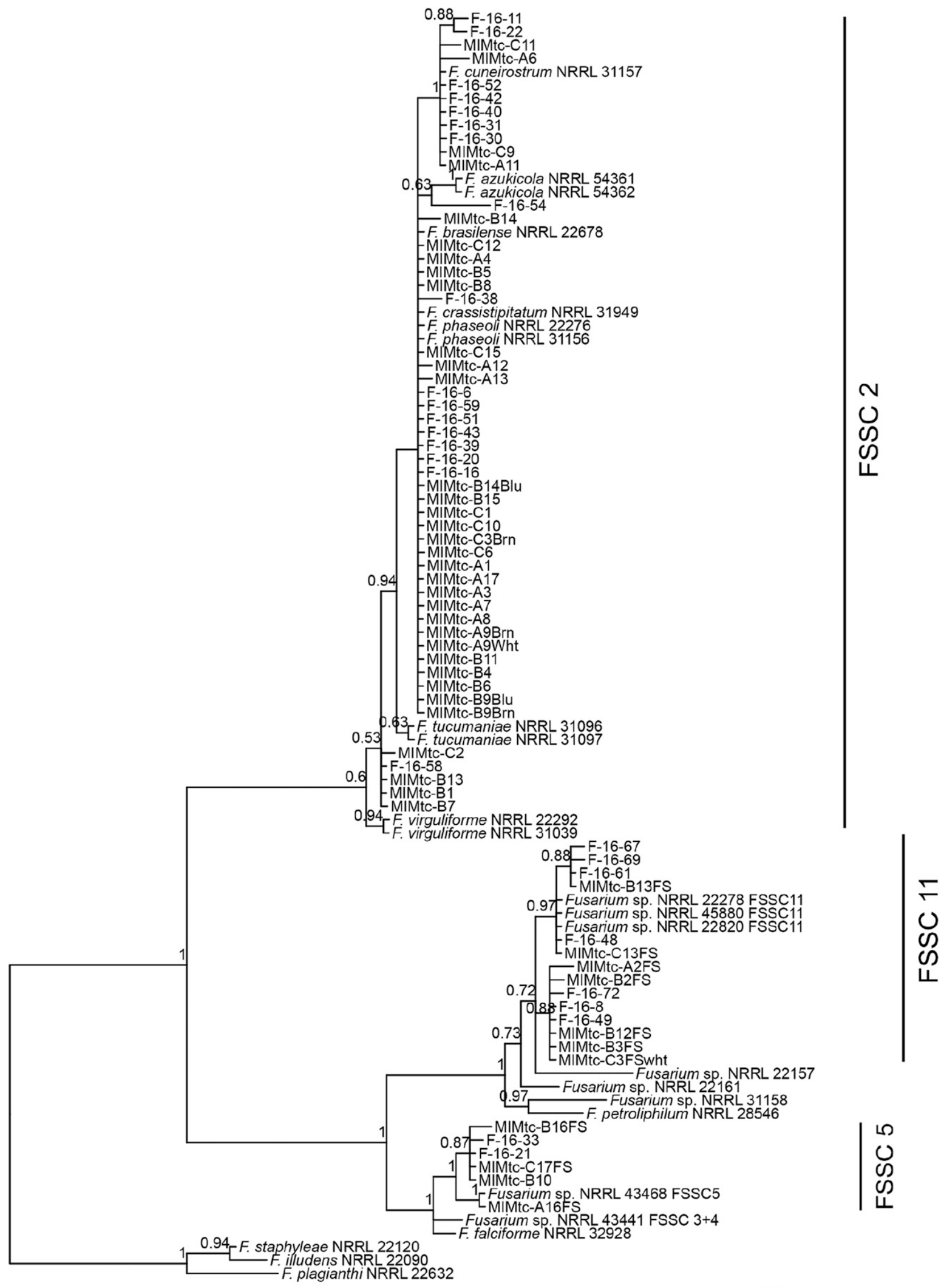

Fig. 1. Bayesian phylogenetic tree of elongation factor-1 $\alpha(E F-1 \alpha)$ sequences showing the phylogenetic relationship of Fusarium brasiliense with other species within the $F$. solani species complex (FSSC). Posterior probabilities are above the nodes. This tree is rooted on the sequences of $F$. staphyleae, $F$. illudens, and $F$. plagianthi from the National Center for Biotechnology Information GenBank. Strains labeled "MIMtc" and "F" were collected from Montcalm, Michigan, in 2014 and 2016, respectively. Strains labeled "NRRL" are from the Agricultural Research Service Culture Collection. 
(Phaseolus vulgaris) in these fields, which is not common for the bulk of soybean production fields in the United States. Both $F$. cuneirostrum and $F$. phaseoli are known to cause root rot of dry bean (Aoki et al. 2003; Henriquez et al. 2014; Jacobs et al. 2018) and strains of these two pathogens ( $F$. cuneirostrum NRRL 31157 and $F$. phaseoli NRRL 31156) used in this study were isolated in previous studies from dry bean in Michigan (Aoki et al. 2005; O'Donnell et al. 2010). In addition, three strains of an unnamed Fusarium sp. grouped with NRRL 22411 isolated from soybean in South Africa (Tewoldemedhin et al. 2017). Tewoldemedhin et al. (2017) proposed that this Fusarium sp. is a fifth species capable of causing soybean SDS. Our results agreed with this suggestion, because the strain Fusarium sp. MIMtc_A4 caused SDS-like symptoms in our growth chamber assay. Members of the same FSSC 5 and FSSC 11 from

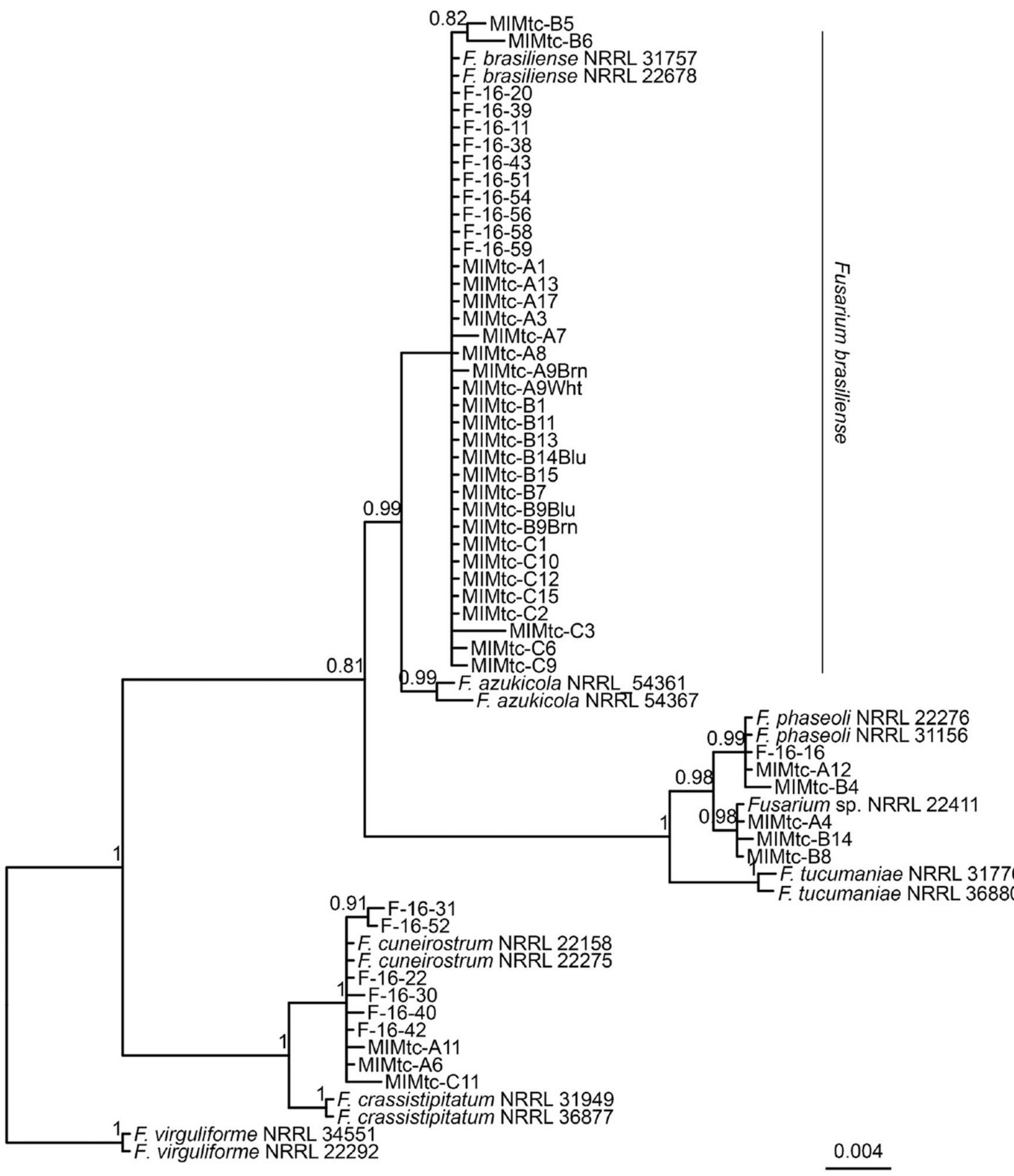

Fig. 2. Bayesian phylogenetic tree of intergenic spacer (IGS) sequences showing the phylogenetic relationship of Fusarium brasiliense with other species within the $F$. solani species complex (FSSC). Posterior probabilities are above the nodes. This tree is rooted on a sequence of $F$. virguliforme from the National Center for Biotechnology Information GenBank. Strains labeled "MIMtc" and "F" were collected from Montcalm, Michigan, in 2014 and 2016, respectively. Strains labeled "NRRL" are from the Agricultural Research Service Culture Collection. 
soybean roots as in the current study were also found in soybean fields in North Dakota and Minnesota (Chitrampalam and Nelson 2016). Members of FSSC 11 have been associated with root rot of pea and soybean (Chitrampalam and Nelson 2016; Snyder and Hansen 1941), and FSSC 5 includes clinically important species (O'Donnell et al. 2008; Zhang et al. 2006). A more thorough evaluation of these species and their possible contribution to soybean root rot and SDS appears to be necessary.

Identifying Fusarium strains to the species level often requires specific molecular markers. EF- $1 \alpha$ sequences can resolve some Fusarium to the species level or at least the species complex level (Aoki et al. 2014; Geiser et al. 2004). However, species within the FSSC clades require additional markers such as the rDNA IGS or RPB2 (O'Donnell et al. 2010). Fusarium species in FSSC clade 2 were able to be classified based on the phylogeny of the rDNA IGS marker (O'Donnell et al. 2010). However, the rDNA IGS sequence works poorly to identify $F$. solani species in other clades of the FSSC. The RPB2 marker has primarily been used for FSSC besides clade 2, particularly for the study of clinical isolates (O'Donnell et al. 2013). Thus, a combination of appropriate markers to conform to the genealogical concordance phylogenetic species recognition concept (Taylor et al. 2000) was used in this study.

Strains within the same Fusarium species caused variable soybean foliar and root disease symptoms. For example, $F$. brasiliense strain MIMtc-C1 caused typical SDS foliar chlorosis but strains MIMtc-A8 and MIMtc-C12 did not, and Fusarium sp. strain MIMtc-A4 caused typical SDS foliar symptoms but strain MIMtc-B8 did not. Intriguingly, the $F$. brasiliense strain causing more root rot induced more foliar symptoms, whereas the Fusarium sp. strain causing more root rot induced fewer SDS foliar symptoms. Within $F$. virguliforme, these SDS foliar symptoms are attributed to fungal phytotoxins such as FvTox1 and FvNIS1 (Chang et al. 2016; Hartman et al. 2015; Pudake et al. 2013). The variation among SDS foliar symptoms in this study might be dependent on the ability of each strain to colonize soybean roots or possibly because of different amounts or types of toxins produced by each strain. In addition to the known SDSinducing species, F. phaseoli strain MIMtc-A12 caused SDS-like foliar symptoms on soybeans under our assay conditions. This agrees with the work of Aoki et al. (2005), who demonstrated that soybean infected by some strains of $F$. phaseoli also exhibit moderate SDS
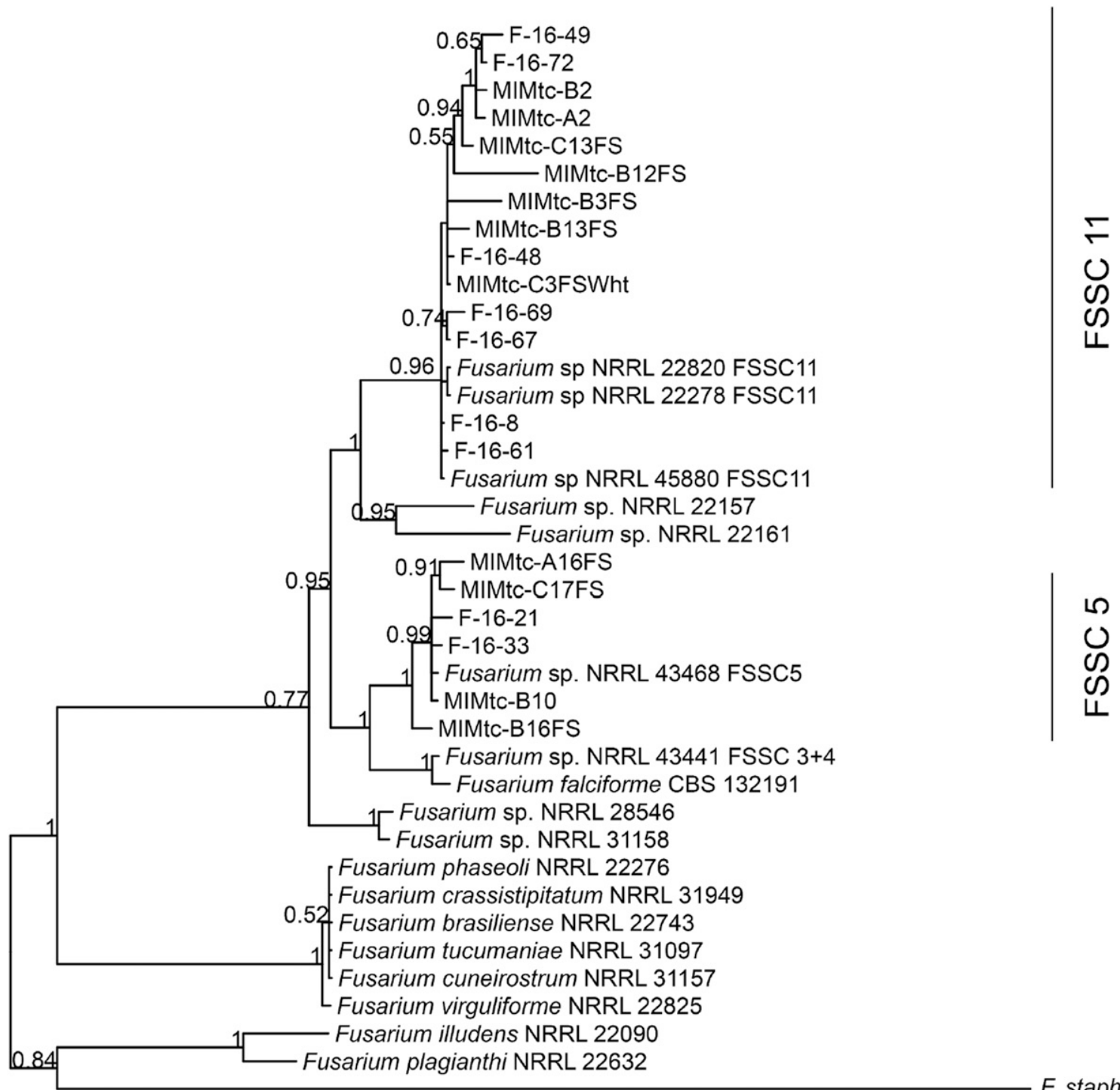

F-16-67

(5)

Fusarium sp NRRL 22278 FSSC11

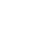

(2)

0

F. staphyleae NRRL 22316

0.02

Fig. 3. Bayesian phylogenetic tree of RNA polymerase II beta subunit (RPB2) sequences showing the phylogenetic relationship of Fusarium species isolated from soybean roots with other species within the $F$. solani species complex (FSSC). Posterior probabilities are above the nodes. This tree is rooted on sequences of $F$. staphyleae, $F$. illudens, and F. plagianthi from the National Center for Biotechnology Information GenBank. Strains labeled "MIMtc" and "F" were collected from Montcalm, Michigan, in 2014 and 2016 , respectively. Strains labeled "NRRL" are from the Agricultural Research Service Culture Collection. 
foliar symptoms. Further studies of phytotoxin production and other virulence factors within the FSSC are required to elucidate the complete mechanisms of foliar and root disease in soybean.

Members of FSSC clade 2 caused more root rot than those of FSSC clade 5; however, members of FSSC clade 5 still produced root rot severity scores greater than $50 \%$ that resulted in root and shoot mass reduction. This is in contrast with the findings of Chitrampalam and
Nelson (2016), who suggested that members of FSSC clade 5 could be facultative pathogens because isolates caused limited lesions or were nonpathogenic on legume species (soybean, pea, dry bean, and lentil). Additional work is needed to explore the contribution of the various members of the FSSC to soybean disease. For example, coinoculation experiments and field trials with different species of FSSC may provide additional insights.
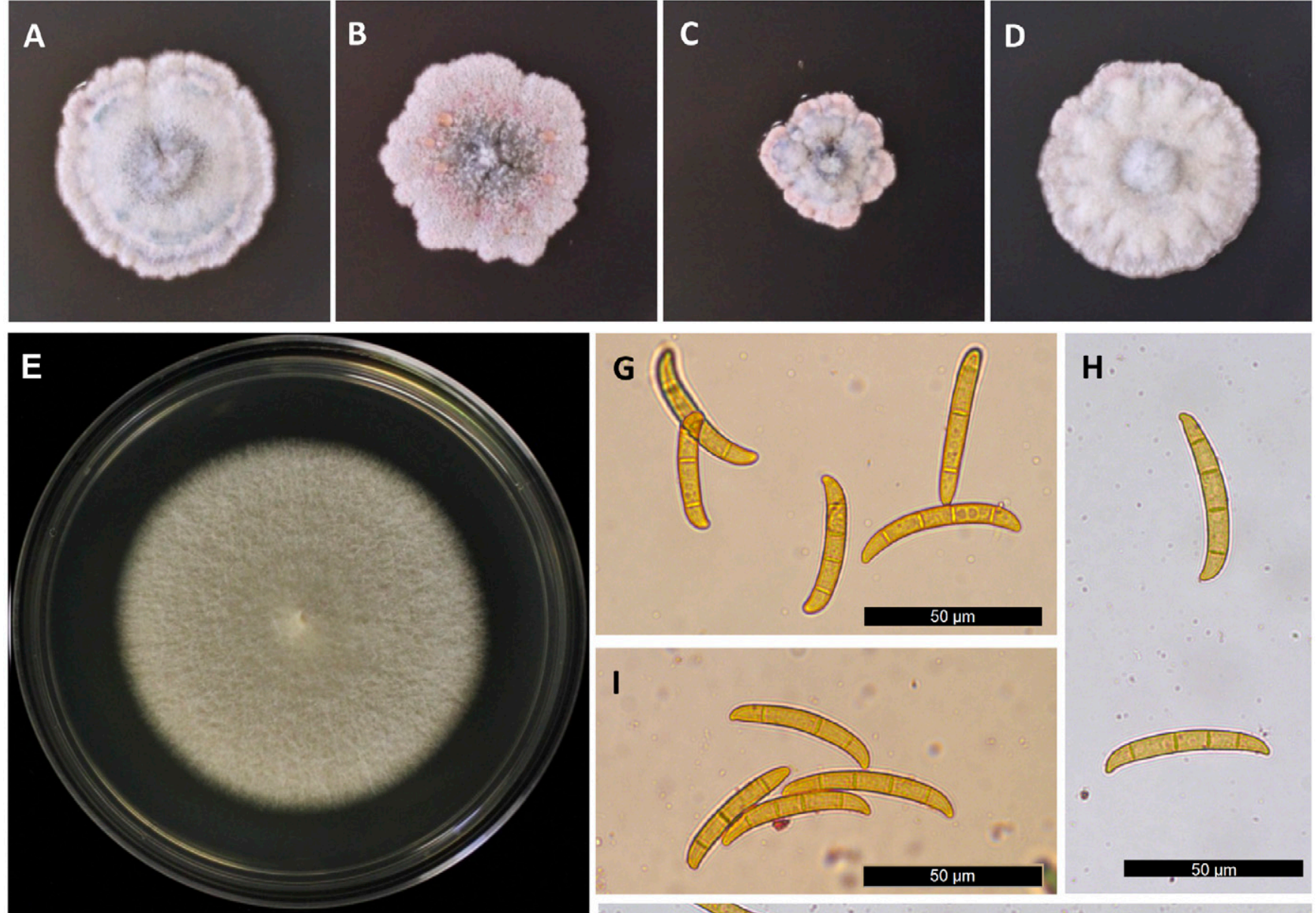

H
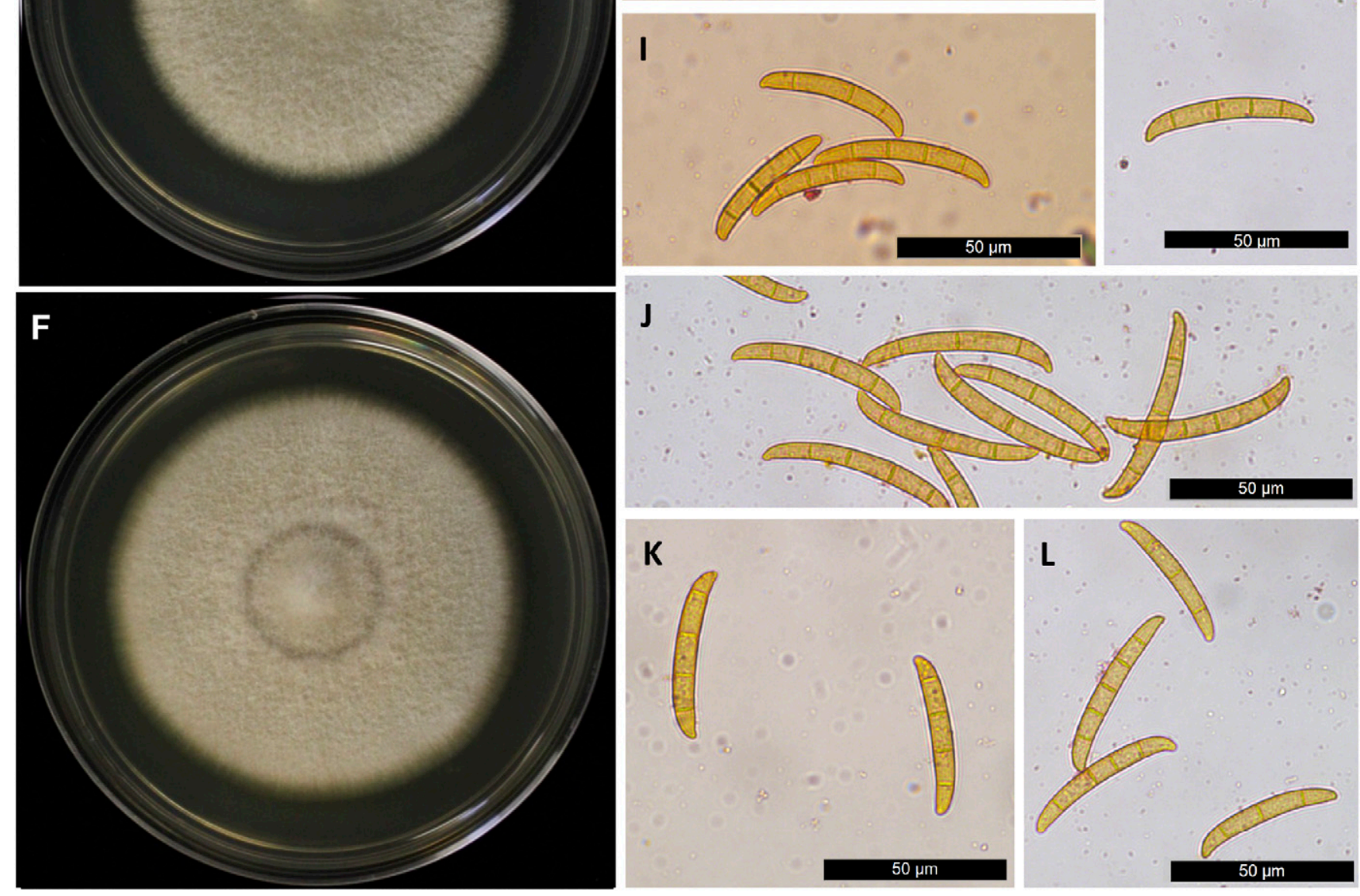

Fig. 4. The colonies and macroconidia of representative Fusarium solani species complex (FSSC) strains isolated from soybean in Michigan. Thirteen-day-old colony of F. brasiliense strain MIMtc-A1 (A), F. cuneirostrum strain MIMtc-C11 (B), F. phaseoli strain MIMtc-A12 (C), and Fusarium sp. strain MIMtc-B8 (D) on potato dextrose agar (PDA). Seven-day-old colony of FSSC 5 strain MIMtc-A16FS (E) and FSSC 11 strain MIMtc-C13FS (F) on PDA. Macroconidia of $F$. brasiliense strain MIMtc-A1 (G), F. cuneirostrum strain MIMtc-C11 (H), F. phaseoli strain MIMtc-A12 (I), Fusarium sp. strain MIMtc-B8 (J), FSSC 5 strain MIMtc-A16FS (K), and FSSC 11 strain MIMtc-C13FS (L) on Spezieller Nährstoffarmer agar and stained with safranin 0. 
Table 2. Pathogenicity of strains of Fusarium species originally isolated from soybean roots in Michigan (MIMtc) or obtained from the Agricultural Research Service Culture Collection (NRRL) on soybean cultivar Sloan in the growth chamber ${ }^{\mathrm{v}}$

\begin{tabular}{|c|c|c|c|c|c|}
\hline Fusarium species $^{w}$ & Strain & $\operatorname{SDS} \operatorname{DIX}(\%)^{\mathrm{x}}$ & Root rot DIX $(\%)^{y}$ & Dry shoot mass (g) & Dry root mass (g) \\
\hline F. brasiliense & NRRL 22678 & $43.8 \mathrm{a}$ & $92.1 \mathrm{ab}$ & $0.25 \mathrm{ef}$ & $0.16 \mathrm{~cd}$ \\
\hline F. tucumaniae & NRRL 31096 & $43.8 \mathrm{a}$ & $89.3 \mathrm{ab}$ & $0.53 \mathrm{c}-\mathrm{f}$ & $0.29 \mathrm{~b}-\mathrm{d}$ \\
\hline F. brasiliense & MIMtc-C1 & $33.8 \mathrm{ab}$ & $90.0 \mathrm{ab}$ & $0.14 \mathrm{f}$ & $0.20 \mathrm{~b}-\mathrm{d}$ \\
\hline F. phaseoli & MIMtc-A12 & $29.4 \mathrm{a}-\mathrm{c}$ & $96.4 \mathrm{a}$ & $0.13 \mathrm{f}$ & $0.06 \mathrm{~d}$ \\
\hline F. virguliforme & NRRL 22292 & $28.8 \mathrm{a}-\mathrm{c}$ & $86.4 \mathrm{ab}$ & $0.92 \mathrm{~b}-\mathrm{d}$ & $0.55 \mathrm{bc}$ \\
\hline Fusarium sp. & MIMtc-A4 & $27.5 \mathrm{a}-\mathrm{d}$ & $80.7 \mathrm{a}-\mathrm{c}$ & $0.67 \mathrm{~b}-\mathrm{f}$ & $0.27 \mathrm{~b}-\mathrm{d}$ \\
\hline F. brasiliense & MIMtc-C9 & $21.9 \mathrm{~b}-\mathrm{e}$ & $83.6 \mathrm{ab}$ & $0.45 \mathrm{c}-\mathrm{f}$ & $0.33 \mathrm{~b}-\mathrm{d}$ \\
\hline Fusarium sp. & MIMtc-B8 & $18.8 \mathrm{~b}-\mathrm{e}$ & $92.1 \mathrm{ab}$ & $0.36 \mathrm{~d}-\mathrm{f}$ & $0.28 \mathrm{~b}-\mathrm{d}$ \\
\hline F. brasiliense & MIMtc-C12 & $18.1 \mathrm{~b}-\mathrm{e}$ & $83.6 \mathrm{ab}$ & $0.74 \mathrm{~b}-\mathrm{f}$ & $0.35 \mathrm{~b}-\mathrm{d}$ \\
\hline F. cuneirostrum & MIMtc-C11 & $18.1 \mathrm{~b}-\mathrm{e}$ & $93.6 \mathrm{ab}$ & 0.24 ef & $0.20 \mathrm{~cd}$ \\
\hline F. cuneirostrum & NRRL 31157 & $14.4 \mathrm{c}-\mathrm{e}$ & $76.4 \mathrm{bc}$ & $1.06 \mathrm{bc}$ & $0.56 \mathrm{bc}$ \\
\hline FSSC 11 & MIMtc-B3FS & $11.9 \mathrm{c}-\mathrm{e}$ & $87.9 \mathrm{ab}$ & $0.85 \mathrm{~b}-\mathrm{e}$ & $0.47 \mathrm{~b}-\mathrm{d}$ \\
\hline Noninoculated control & & $11.9 \mathrm{c}-\mathrm{e}$ & $21.4 \mathrm{e}$ & $2.18 \mathrm{a}$ & $1.01 \mathrm{a}$ \\
\hline F. brasiliense & MIMtc-A8 & $11.3 \mathrm{c}-\mathrm{e}$ & $82.9 \mathrm{ab}$ & $0.67 \mathrm{~b}-\mathrm{f}$ & $0.42 \mathrm{~b}-\mathrm{d}$ \\
\hline FSSC 5 & MIMtc-C17FS & $11.3 \mathrm{c}-\mathrm{e}$ & $50.0 \mathrm{~d}$ & $1.25 \mathrm{~b}$ & $0.64 \mathrm{ab}$ \\
\hline F. phaseoli & NRRL 31156 & $9.4 \mathrm{de}$ & $81.4 \mathrm{ab}$ & $0.69 \mathrm{~b}-\mathrm{f}$ & $0.41 \mathrm{~b}-\mathrm{d}$ \\
\hline FSSC 11 & MIMtc-C13FS & $8.8 \mathrm{de}$ & $48.6 \mathrm{~d}$ & $1.16 \mathrm{~b}$ & $0.47 \mathrm{~b}-\mathrm{d}$ \\
\hline FSSC 5 & MIMtc-A16FS & $6.9 \mathrm{e}$ & $62.1 \mathrm{~cd}$ & $0.97 \mathrm{~b}-\mathrm{d}$ & $0.43 \mathrm{~b}-\mathrm{d}$ \\
\hline \multicolumn{6}{|l|}{$P$ value } \\
\hline Species & & $<0.0001$ & $<0.0001$ & $<0.0001$ & $<0.0001$ \\
\hline Strain $(\text { species } \times \text { replicate })^{\mathrm{z}}$ & & $<0.0001$ & $<0.0001$ & $<0.0001$ & $<0.0001$ \\
\hline
\end{tabular}

${ }^{v}$ Plants were examined for both foliar symptoms typical of sudden death syndrome (SDS) and root rot. Means followed by the same letter are not significant $(P<0.05)$ according to Fisher's protected least significant difference test.

${ }^{\mathrm{w}}$ Fusarium sp. indicates that the species has not yet been named. FSSC $=F$. solani species complex.

$\mathrm{x}$ The SDS disease severity index (DIX) that accounted for both disease severity (DS) and disease incidence (DI) was calculated as follows: DIX $=$ DI $\times($ DS/ maximum disease score) $\times 100$.

y The root rot DIX was calculated as follows: DIX $=$ DI $\times($ DS/maximum disease score $) \times 100$.

${ }^{\mathrm{z}}$ Strain nested within the interaction between species and replicate was the random effect.

To date, the management of SDS in the United States has focused on $F$. virguliforme. These newly reported Fusarium species may need to be included for phenotyping of disease severity to improve soybean resistance to both foliar SDS and root rot. In addition, the efficacy of the recently labeled fungicide seed treatment, fluopyram (succinate dehydrogenase inhibitor), for SDS management (Kandel et al. 2016, 2018) should be determined for these additional species. The effect of crop rotation should also be determined, because some of these species are reported to colonize or be pathogenic on various crops (Kolander et al. 2012). It has been demonstrated that singleyear corn rotations offer little value to reducing subsequent SDS pressure when $F$. virguliforme is the primary pathogen present; however, extended rotations with diversified crop systems coupled with the use of organic matter amendments have demonstrated promise (Leandro et al. 2018). Our study indicates that rotating to dry bean may favor other members of the FSSC. We hypothesize that this may be a reason why $F$. brasiliense is more common in South America, where dry bean is a major crop (Broughton et al. 2003). In addition, current qPCR assays specifically designed for the detection of $F$. virguliforme are specific only to $F$. virguliforme and other SDScausing pathogens cannot be detected. Therefore, diagnostic assays that are specific to or can distinguish between Fusarium SDS- and root rot-causing pathogens are needed.

\section{Acknowledgments}

Some reference DNA sequence files were kindly provided by Dr. Kerry O’Donnell.

\section{Literature Cited}

Aoki, T., O'Donnell, K., and Geiser, D. M. 2014. Systematics of key phytopathogenic Fusarium species: Current status and future challenges. J. Gen. Plant Pathol. 80:189-201.

Aoki, T., O’Donnell, K., Homma, Y., and Lattanzi, A. R. 2003. Sudden-death syndrome of soybean is caused by two morphologically and phylogenetically distinct species within the Fusarium solani species complex $-F$. virguliforme in North America and F. tucumaniae in South America. Mycologia 95: $660-684$
Aoki, T., O'Donnell, K., and Scandiani, M. M. 2005. Sudden death syndrome of soybean in South America is caused by four species of Fusarium: Fusarium brasiliense sp. nov., $F$. cuneirostrum sp. nov., $F$. tucumaniae, and $F$. virguliforme. Mycoscience 46:162-183.

Aoki, T., Scandiani, M., and O'Donnell, K. 2012. Phenotypic, molecular phylogenetic, and pathogenetic characterization of Fusarium crassistipitatum sp. nov., a novel soybean sudden death syndrome pathogen from Argentina and Brazil. Mycoscience 53:167-186.

Broughton, W. J., Hernández, G., Blair, M., Beebe, S., Gepts, P., and Vanderleyden, J. 2003. Beans (Phaseolus spp.) - model food legumes. Plant Soil 252:55-128.

Chang, H. X., Domier, L. L., Radwan, O., Yendrek, C. R., Hudson, M. E., and Hartman, G. L. 2016. Identification of multiple phytotoxins produced by Fusarium virguliforme including a phytotoxic effector (FvNIS1) associated with sudden death syndrome foliar symptoms. MPMI 29:96-108.

Chilvers, M. I., and Brown-Rytlewski, D. E. (2010) First report and confirmed distribution of soybean sudden death syndrome caused by Fusarium virguliforme in southern Michigan. Plant Dis. 94:1164.

Chilvers, M. I., Byrne, J., and MacKellar, B. 2016 Soybean sudden death syndrome spreading, now confirmed in Livingston County. Michigan State University Extension. Retrieved 21 June 2018 from http://msue.anr msu.edu/news/soybean_sudden_death_syndrome_spreading_now_confirmed_ in_livingston_county.

Chitrampalam, P., and Nelson, B., Jr. 2016. Multilocus phylogeny reveals an association of agriculturally important Fusarium solani species complex (FSSC) 11, and clinical important FSSC 5 and FSSC $3+4$ with soybean roots in the north central United States. Antonie van Leeuwenhoek 109: 335-347.

Edgar, R. C. 2004. MUSCLE: Multiple sequence alignment with high accuracy and high throughput. Nucleic Acids Res. 32:1792-1797.

Geiser, D. M., Jimenez-Gasco, M. D., Kang, S. C., Makalowska, I., Veeraraghavan, N., Ward, T. J., Zhang, N., Kuldau, G. A., and O'Donnell, K. 2004. FUSARIUM-ID v. 1.0: A DNA sequence database for identifying Fusarium. Eur. J. Plant Pathol. 110:473-479.

Hartman, G. L., Chang, H. X., and Leandro, L. F. 2015. Research advances and management of soybean sudden death syndrome. Crop Prot. 73:60-66.

Henriquez, M. A., McLaren, D. L., Conner, R. L., Balasubramanian, P. M., Chang, K. F., Hwang, S. F., and Strelkov, S. E. 2014. First report of Fusarium cuneirostrum causing root rot disease in dry bean (Phaseolus vulgaris) in Canada. Plant Dis. 98:278.

Jacobs, J. L., Oudman, K., Sang, H., and Chilvers, M. I. 2018. First report of Fusarium brasiliense causing root rot of dry bean in Michigan. Plant Dis. 102:2035. 
Kandel, Y. R., McCarville, M. T., Adee, E. A., Bond, J. P., Chilvers, M. I., Conley, S. P., Giesler, L. J., Kelly, H. M., Malvick, D. K., Mathew, F. M., Rupe, J. C., Sweets, L. E., Tenuta, A. U., Wise, K. A., and Mueller, D. S. 2018. Benefits and profitability of fluopyram-amended seed treatments for suppressing sudden death syndrome and protecting soybean yield: A meta-analysis. Plant Dis. 102:1093-1100.

Kandel, Y. R., Wise, K. A., Bradley, C. A., Chilvers, M. I., Tenuta, A. U., and Mueller, D. S. 2016. Fungicide and cultivar effects on sudden death syndrome and yield of soybean. Plant Dis. 100:1339-1350.

Kolander, T. M., Bienapfl, J. C., Kurle, J. E., and Malvick, D. K. 2012. Symptomatic and asymptomatic host range of Fusarium virguliforme, the causal agent of soybean sudden death syndrome. Plant Dis. 96:1148-1153.

Leandro, L. F. S., Eggenberger, S., Chen, C., Williams, J., Beattie, G. A., and Liebman, M. 2018. Cropping system diversification reduces severity and incidence of soybean sudden death syndrome caused by Fusarium virguliforme. Plant Dis. 102:1748-1758.

Leslie, J. F., and Summerell, B. A., eds. 2006. The Fusarium Laboratory Manual, 1st ed. Blackwell Publishing Ltd, Oxford, UK.

Nakajima, T., Mitsueda, T., and Charchar, M. 1993. Occurrence of soybean sudden-death syndrome caused by Fusarium solani in Brazil. Page P79 in: Proceedings of the Seventh International Fusarium Workshop. Pennsylvania State University, University Park, PA.

National Agricultural Statistics Service. 2018. Crop production, released July 12, 2018. United States Department of Agriculture, Washington, DC. Available at: https://usda.library.cornell.edu/concern/publications/tm70mv177

O'Donnell, K. 2000. Molecular phylogeny of the Nectria haematococca-Fusarium solani species complex. Mycologia 92:919-938.

O’Donnell, K., Kistler, H. C., Cigelnik, E., and Ploetz, R. C. 1998. Multiple evolutionary origins of the fungus causing Panama disease of banana: Concordant evidence from nuclear and mitochondrial gene genealogies. Proc. Natl. Acad. Sci. USA 95:2044-2049.

O’Donnell, K., Rooney, A. P., Proctor, R. H., Brown, D. W., McCormick, S. P., Ward, T. J., Frandsen, R. J., Lysoe, E., Rehner, S. A., Aoki, T., Robert, V. A., Crous, P. W., Groenewald, J. Z., Kang, S., and Geiser, D. M. 2013. Phylogenetic analyses of RPB1 and RPB2 support a middle Cretaceous origin for a clade comprising all agriculturally and medically important fusaria. Fungal Genet. Biol. 52:20-31.

O’Donnell, K., Sarver, B. A. J., Brandt, M., Chang, D. C., Noble-Wang, J., Park, B. J., Sutton, D. A., Benjamin, L., Lindsley, M., Padhye, A., Geiser, D. M., and Ward, T. J. 2007. Phylogenetic diversity and microsphere array-based genotyping of human pathogenic fusaria, including isolates from the multistate contact lens-associated U.S. keratitis outbreaks of 2005 and 2006. J. Clin. Microbiol. 45:2235-2248.

O’Donnell, K., Sink, S., Scandiani, M., Luque, A., Colletto, A., Biasoli, M., Lenzi, L., Salas, G., González, V., and Ploper, L. 2010. Soybean sudden death syndrome species diversity within North and South America revealed by multilocus genotyping. Phytopathology 100:58-71.

O’Donnell, K., Sutton, D. A., Fothergill, A., McCarthy, D., Rinaldi, M. G., Brandt, M. E., Zhang, N., and Geiser, D. M. 2008. Molecular phylogenetic diversity, multilocus haplotype nomenclature, and in vitro antifungal resistance within the Fusarium solani species complex. J. Clin. Microbiol. 46:2477-2490.

Peever, T. L., Canihos, Y., Olsen, L., Ibañez, A., Liu, Y. C., and Timmer, L. W. 1999. Population genetic structure and host specificity of Alternaria spp. causing brown spot of minneola tangelo and rough lemon in Florida. Phytopathology 89:851-860.
Pudake, R. N., Swaminathan, S., Sahu, B. B., Leandro, L. F., and Bhattacharyya, M. K. 2013. Investigation of the Fusarium virguliforme fvtox 1 mutants revealed that the FvTox 1 toxin is involved in foliar sudden death syndrome development in soybean. Curr. Genet. 59:107-117.

Reeb, V., Lutzoni, F., and Roux, C. 2004. Contribution of RPB2 to multilocus phylogenetic studies of the Euascomycetes (Pezizomycotina, Fungi) with special emphasis on the lichen-forming Acarosporaceae and evolution of polyspory. Mol. Phylogenet. Evol. 32:1036-1060.

Ronquist, F., and Huelsenbeck, J. P. 2003. MrBayes 3: Bayesian phylogenetic inference under mixed models. Bioinformatics 19:1572-1574.

Roy, K. W., Rupe, J. C., Hershman, D. E., and Abney, T. S. 1997. Sudden death syndrome of soybean. Plant Dis. 81:1100-1111.

Rupe, J. C., Correll, J. C., and Guerber, J. C. 2001. Differentiation of the sudden death syndrome pathogen of soybean, Fusarium solani f.sp. glycines, from other isolates of $F$. solani based on cultural morphology, pathogenicity, and mitochondrial DNA restriction fragment length polymorphisms. Can. J. Bot. 79:829-835.

Schliep, K. P. 2011. phangorn: Phylogenetic analysis in R. Bioinformatics 27: 592-593.

Shapiro, S. S., and Wilk, M. B. 1965. An analysis of variance test for normality (complete samples). Biometrika 52:591-611.

Snyder, W. C., and Hansen, H. N. 1941. The species concept in Fusarium with reference to section Martiella. Am. J. Bot. 28:738-742.

Tamura, K., Stecher, G., Peterson, D., Filipski, A., and Kumar, S. 2013. MEGA6: Molecular Evolutionary Genetics Analysis version 6.0. Mol. Biol. Evol. 30: $2725-2729$

Taylor, J. W., Jacobson, D. J., Kroken, S., Kasuga, T., Geiser, D. M., Hibbett, D. S., and Fisher, M. C. 2000. Phylogenetic species recognition and species concepts in fungi. Fungal Genet. Biol. 31:21-32.

Tewoldemedhin, Y. T., Lamprecht, S. C., Geldenhuys, J. J., and Kloppers, F. J. 2014. First report of soybean sudden death syndrome caused by Fusarium virguliforme in South Africa. Plant Dis. 98:569.

Tewoldemedhin, Y. T., Lamprecht, S. C., Vaughan, M. M., Doehring, G., and O'Donnell, K. 2017. Soybean SDS in South Africa is caused by Fusarium brasiliense and a novel undescribed Fusarium sp. Plant Dis. 101:150-157.

Wang, J., and Chilvers, M. I. 2016. Development and characterization of microsatellite markers for Fusarium virguliforme and their utility within clade 2 of the Fusarium solani species complex. Fungal Ecol. 20:7-14.

Wang, J., Jacobs, J. L., Byrne, J. M., and Chilvers, M. I. 2015. Improved diagnoses and quantification of Fusarium virguliforme, causal agent of soybean sudden death syndrome. Phytopathology 105:378-387.

Wang, J., Jacobs, J. L., Roth, M. G., and Chilvers, M. I. 2019. Temporal dynamics of Fusarium virguliforme colonization of soybean roots. Plant Dis. 103:19-27.

Westphal, A., Abney, T. S., Xing, L. J., and Shaner, G. E. 2008. Sudden death syndrome of soybean. Plant Health Instructor.

Yu, G., Smith, D. K., Zhu, H., Guan, Y., and Lam, T. T. Y. 2017. ggtree: An R package for visualization and annotation of phylogenetic trees with their covariates and other associated data. Methods Ecol. Evol. 8:28-36.

Zhang, N., O'Donnell, K., Sutton, D. A., Nalim, F. A., Summerbell, R. C., Padhye, A. A., and Geiser, D. M. 2006. Members of the Fusarium solani species complex that cause infections in both humans and plants are common in the environment. J. Clin. Microbiol. 44:2186-2190. 